\title{
ANÁLISE DO EFEITO DAS PRÁTICAS SOCIOTÉCNICAS NO DESEMPENHO EM QUALIDADE E SAÚDE DOS COLABORADORES EM EMPRESAS COM IMPLEMENTAÇÃO LEAN*
}

\author{
Guilherme Luz Tortorella** \\ Diego Castro Fettermann **:* \\ Lizandra Garcia Lupi Vergara*****
}

doi: 10.11144/Javeriana.cao.31-56.aepsd. El artículo se recibió el 21/11/2017 y se aprobó 09/05/2018.

Sugerencia de citación: Tortorella, G. L., Fettermann, D. C. e Vergara, L. G. L. (2018). Análise do efeito das práticas sociotécnicas no desempenho em qualidade e saúde dos colaboradores em empresas com implementação Lean. Cuadernos de Administración. 31(56), 31-54. http://dx.doi.org/10.11144/Javeriana.cao.31-56.aepsd.

* * Doctor en Ingeniería de Producción de la Universidade Federal do Rio Grande do Sul, Porto Alegre, Brasil, 2012. Profesor del Centro Tecnológico, Universidade Federal de Santa Catarina, Florianopolis, Brasil. E-mail: g.tortorella@ufsc.br

* * * Doctor en Ingeniería de Producción de la Universidade Federal do Rio Grande do Sul, Porto Alegre, Brasil, 2013. Profesor del Centro Tecnológico, Universidade Federal de Santa Catarina, Florianopolis, Brasil. E-mail: d.fettermann@ufsc.br

**** Doctor en Ingeniería de Producción de la Universidade Federal de Santa Catarina, Florianópolis, Brasil. Profesor del Centro Tecnológico, Universidade Federal de Santa Catarina, Florianopolis, Brasil. E-mail: l.vergara@ufsc.br 


\author{
Análise do efeito das \\ práticas sociotécnicas no \\ desempenho em qualidade \\ e saúde dos colaboradores \\ em empresas com \\ implementação Lean
}

\section{ResUmo}

A utilização de práticas sociotécnicas é mencionada como um direcionador da satisfação dos colaboradores dentro da organização. Este artigo tem por objetivo identificar o efeito moderador da aplicação de práticas just-in-time sobre a relação entre as práticas sociotécnicas e o desempenho de qualidade de saúde dos colaboradores em empresas com implementação Lean. Foi desenvolvida uma pesquisa com 144 empresas do sul do Brasil em processo de implementação Lean. Os resultados indicam que as práticas sociotécnicas apresentam relação significativa com o desempenho da empresa em qualidade e saúde dos colaboradores, e a implementação de práticas just-in-time não prejudica o desempenho desses indicadores nas empresas.

Palavras-chave: práticas sociotécnicas, qualidade, saúde dos colaboradores, just-in-time.

Códigos JEL: J21, J81, L69.

Análisis del efecto de las prácticas sociotécnicas en el desempeño en calidad y salud de los colaboradores en empresas con implementación Lean

\section{RESUMEN}

El uso de prácticas socio-técnicas se menciona como un indicador de satisfacción de los colaboradores dentro de la organización. Este artículo tiene como propósito identificar el efecto moderador de la aplicación de prácticas just-in-time sobre la relación entre las prácticas socio-técnicas y el desempeño de calidad de salud de los colaboradores en empresas con implementación Lean. Se desarrolló una investigación con 144 empresas del sur de Brasil en proceso de implementación Lean. Los resultados indican que las prácticas socio-técnicas presentan relación significativa con el desempeño de la empresa en calidad y salud de los colaboradores, y la implementación de prácticas just-in-time no perjudica el desempeño de estos indicadores en las empresas.

Palabras clave: prácticas socio-técnicas, calidad, salud de los colaboradores, just-in-time. Códigos JEL: J21, J81, L69.
Analysis of the sociotechnical practices effect on performance of quality and workers' health in companies undergoing lean implementation

\begin{abstract}
The use of Socio-technical practices theory is mentioned as a satisfaction indicator of the work force within an organization. The purpose of this article is to identify the moderating effect at applying just-in-time practices on the relationship between socio-technical practices and the performance of the employees' health quality in such companies. We carried out a survey with 144 different companies from Southern Brazil that are undergoing a lean implementation. Results indicate that Socio-technical practices have a positive significant relationship with such performance, and the concurrent adoption of just-in-time does not undermine quality and workers' health in the companies.
\end{abstract}

Keywords: Socio-technical practices; Quality, Workers' health, Just-in-time. JEL Code: J21, J81, L69. 


\section{Introdução}

A organização do trabalho tem apresentado alterações sem precedentes, em parte como resultado do crescimento de variedade de novas práticas e técnicas de gestão (Zare et ál., 2016). Neste sentido, a teoria sóciotécnica (ST) enfatiza a noção de que o desempenho de novos sistemas pode ser melhorado a partir de uma abordagem sistêmica. A ST menciona que para a organização ser gerenciada de forma satisfatória, o social e o técnico devem ser tratados como aspectos interdependentes de um sistema de trabalho (Clegg, 2000; Koukoulaki, 2014). Assim, a teoria ST tem sido definida como uma teoria de projeto e qualidade de vida no trabalho, cuja perspectiva incorpora explicitamente a ideia de que todos os aspectos de um sistema estão interconectados, e que devem ser projetados conjuntamente (Molleman e Broekhuis, 2001; Kang et ál., 2016). No entanto, muitas organizações não apresentam uma abordagem integrada para o projeto de novos processos ou sua melhoria, tanto nos aspectos sociais quanto técnicos. Em geral, as iniciativas existentes consideram a tecnologia como uma informação; por en quanto o projeto do processo deve ser realizado para se adaptar a tecnologia (Fettermann et ál., 2018). Este fato pode implicar uma implementação parcial ou restrita de práticas ST em sua cobertura e perspectiva, o que prejudica a aplicação plena do processo ST com todos seus benefícios (Eklund, 2000; Carayon, 2006; Koukoulaki, 2010).

As pesquisas relacionadas com práticas ST têm por muito tempo focado no trabalho de processos produzidos em grande escala. A implementação de princípios e práticas do Lean Manufacturing (LM) tornou-se popular entre as indústrias transformadoras, serviços e áreas comerciais (Womack e Jones, 2010). A câmbio no sentido de uma implementação LM implica um refinamento dos sistemas de produção de massa tradicionais, eliminando desperdícios e respondendo a mudanças imprevistas no nível operacional (Liker, 2006; Liker e Hoseus, 2008). A implementação do LM significa uma abordagem sistemática de vários métodos e práticas de gestão, que apresenta o elemento humano como um fator fundamental para a melhoria contínua (Seppälä e Klemola, 2004; Bäckstrand et ál., 2013). Para conseguir um sistema verdadeiramente enxuto, pesquisas anteriores indicam que as práticas LM exigem cada vez mais da força de trabalho a fim de atingir níveis mais elevados de qualidade e flexibilidade com custos mais baixos (Plonka, 1997; Guimarães et ál., 2015), levando os recursos musculares, cognitivos e emocionais do indivíduo ao limite (Hoffmeister et ál., 2015). Ao mesmo tempo, promove uma variedade de tarefas, a segurança do emprego, os incentivos financeiros, o desenvolvimento e a utilização de habilidades e conhecimentos, e o conhecimento do desempenho organizacional (Saurin e Ferreira, 2009; Toralla et ál., 2012), corroborando com os objetivos das práticas ST. 
Neste sentido, é necessária uma visão holística da influência da implementação lean a fim de identificar a adoção e o apoio simultâneo de práticas LM com o efeito das práticas de ST para melhorar o desempenho operacional (Tortorella et ál., 2016b). Além disso, a investigação sobre a relação entre as práticas de ST e o desempenho operacional em contextos organizacionais submetidos a uma implementação LM têm sido prejudicada pelas insuficiências dos estudos anteriores, tanto do ponto de vista teórico como metodológico (Wilson, 2005; Arezes et ál., 2015). Na verdade, poucas organizações estudam suas operações em detalhe, e os que tentam fazê-lo, normalmente os abordam a partir de uma perspectiva limitada (Hagg, 2003; Arezes et ál., 2010).

Neste contexto, o presente estudo tem como objetivo verificar o efeito moderador de práticas enxutas sobre a relação entre as práticas de ST e o desempenho de qualidade e saúde dos trabalhadores. Foi realizado um levantamento com 144 diferentes empresas de manufatura do sul do Brasil que são submetidas a uma implementação lean. Os entrevistados foram convidados a preencher três questionários sequenciais na pesquisa: (i) detalhes sobre as variáveis contextuais apontadas na literatura como influentes para a melhoria de desempenho, que são ainda consideradas como variáveis de controle; (ii) o nível de implementação de práticas de ST e LM; e (iii) o nível de melhoria de desempenho observada ao longo dos últimos cinco anos em relação à qualidade e saúde. Nosso estudo preenche uma lacuna observada na literatura sobre a integração de práticas LM em organizações que têm amplamente execução práticas ST, uma vez que permite a identificação dos efeitos de tal interação na qualidade e desempenho da saúde dos trabalhadores.

Mais especificamente, examinamos o efeito de um conjunto de práticas lean reconhecidos pela redução de estoques e o cuidado com a superprodução, denotado como Just-in-Time (JIT). 0 JIT tem sido extensivamente estudado e empiricamente validado na literatura lean (Shah e Ward, 2003; Shah e Ward, 2007; Netland e Ferdows, 2014; Marodin et ál., 2016), e evidências existentes apontam que a combinação de práticas enxutas corroboram para a construção de um melhor desempenho operacional. No entanto, poucas evidências apoiam que a introdução do JIT pode levantar outras questões operacionais, tais como um aumento na pressão emocional e cognitiva sobre os trabalhadores, levando a diferentes problemas que possam estar ocultos ou mitigados em contextos da produção em massa tradicionais. Neste sentido, não há evidências empíricas que investigam o efeito da moderação de tais práticas sobre a relação entre as práticas de ST e desempenho operacional. A literatura sobre o tema é ainda escassa e sugere associações contraditórias entre as práticas de LM e ST para melhoria do desempenho. Postulamos 
dois construtos de práticas inter-relacionadas e internamente consistentes sobre ST, que foram conceitualmente propostas na literatura; estes são: Projeto de Trabalho (PT) e as Práticas Organizacionais (P0). Nós empiricamente validamos esses construtos e mais tarde investigamos seus efeitos simultâneos sobre a qualidade (sucata e retrabalho) e saúde dos trabalhadores.

Além da contribuição teórica, nossa pesquisa fornece implicações gerenciais que podem apoiar líderes e profissionais a compreender melhor a necessidade e as vantagens de uma implementação simultânea de práticas LM e ST entre as organizações. Além disso, identificar o efeito da interação dessas práticas pode contribuir para abordar as atividades de melhoria específica e reforçar as práticas de gestão que melhor suportam os resultados de desempenho esperados sobre a qualidade e saúde dos trabalhadores. Devido à natureza exploratória desta pesquisa, é desenvolvido um conjunto de proposições em vez de hipóteses formais. Portanto, algumas propostas são investigadas a fim de obter uma compreensão mais clara em torno do objeto e permitir uma melhor declaração sobre as condições de contorno que circundam o problema.

0 artigo está estruturado da seguinte forma: a seção 1 apresenta o fundamentação teórica e as proposições desenvolvidas para alcançar o nosso objetivo de pesquisa. A seção 2 descreve como foi realizado o método de pesquisa através do levantamento de empresas brasileiras e suas práticas de ST e desempenho, construindo procedimentos de validação e testes de nossas proposições usando mínimos quadrados ordinários. Apresentamos os resultados de nossa pesquisa na seção 3 e discussões na seção 4. E o artigo fecha com as conclusões e considerações finais.

\section{Literatura e Proposições}

\subsection{Práticas ST e seus efeitos sobre a saúde e qualidade de trabalhadores}

Qualquer empresa de sucesso deve possuir uma organização de trabalho eficaz e eficiente a nível de toda a gestão, a fim de equilibrar as demandas de trabalho e as condições da força de trabalho e posteriormente, estabelecer as melhores práticas ST propícias para o máximo da saúde humana, produtividade e da qualidade do trabalho (Karwowski et ál., 1994;. Genaidy e Karwowski, 2003; Jaworek et ál., 2010). Práticas ST visam melhorar o ambiente de trabalho em termos de suas exigências físicas, cognitivas e organizacionais, permitindo uma melhor condição de trabalho para todos os funcionários e, portanto, o que implica um melhor desempenho operacional. Em última análise, a prestação de um 
bom clima organizacional está associada à motivação dos funcionários e reforça uma melhor comunicação interpessoal e um bom relacionamento entre os funcionários e seus gerentes (Blaikie et ál., 2014). Portanto, os conceitos subjacentes às práticas ST podem ser considerados no planejamento e na execução das atividades operacionais, a fim de estabelecer condições adequadas e melhores resultados na interação entre trabalhadores e o ambiente de trabalho (Beevis, 2003; Ferreira e Gurgueira, 2013).

Pesquisas anteriores sobre práticas ST possuem evidências de que algumas das interdependências de práticas ST podem não ser tão evidentes durante o projeto do sistema conforme o esperado. Pode haver consequências não intencionais de várias iniciativas de mudança, cujos impactos são difíceis de prever e algumas dessas consequências só podem tornar-se óbvias quando o sistema estiver em operação (Molleman e Broekhuis, 2001; Carayon, 2006; Dixon et ál., 2009). Neste sentido, a compreensão adequada dos efeitos da implementação de práticas ST no desempenho operacional, ainda precisa sofrer um maior aprofundamento de investigação. A implementação de práticas ST implicam mudanças nas responsabilidades organizacionais, em que a separação entre o planejamento que faz parte do sistema tende a diminuir (Eklund, 2000; Dul e Neumann, 2009; Koukoulaki, 2010). Por isso, as empresas são levadas a desafiar os seus funcionários a assumir a propriedade quando e onde for necessário; aprendendo com outras equipes, tanto dentro como fora da empresa, o que é supostamente predominante (Joseph, 2003; Thun et ál., 2011; Tortorella et ál., 2015).

Com base em uma revisão da literatura, 18 práticas destacam-se como as práticas ST mais frequentes em um nível de adoção da gestão, como mostrado na Tabela 1. Destas 18, as práticas de "procura por um bom clima organizacional" e "pesquisa sobre a saúde e segurança dos trabalhadores" parecem ser as mais citadas na literatura. Estas práticas são normalmente associadas com os princípios organizacionais e objetivos estratégicos que são implantados nas rotinas da gestão (Azadeh et ál., 2014;. Hoffmeister et ál., 2015). Estas rotinas gerenciais visam reforçar e fornecer orientação adequada para os gestores e suas equipes com relação a aspectos técnicos, organizacionais e humanos em uma base diária para todas as atividades produtivas de dentro da empresa (Karsh et ál., 2014). É possível notar que alguns pesquisadores também relacionam essas práticas com padrões de comportamento esperado sob o ambiente de trabalho (Asadzadeh et ál., 2013; Guimarães et ál., 2014). Em oposição, “gestão da rotatividade de pessoas” parece ser uma prática que ainda não foi totalmente adotada e em particular, reconhecida como benéfica para a implementação de práticas ST. No entanto, estudos que evidenciam sua importância relatam vantagens na sua aprovação a partir da perspectiva 
ST (Pavlovic-Veselinovic et ál., 2016; Kang et ál. (2016). Em geral, estas 18 práticas ST têm sido consistentemente evidenciadas na literatura e podem representar, para a finalidade deste estudo, a implementação de práticas de ST dentro de uma empresa.

Destas, foram selecionados práticas inter-relacionadas para combinar em dois principais construtos de práticas associadas a PT e P0. 0 construto PT pode compreender práticas que corroboram para melhorar a ergonomia e condições de saúde da tarefa de trabalho, incluindo outros elementos, como ferramentas e o ambiente físico do trabalho (Clegg, 2000; Arezes et ál., 2010). Os efeitos da PT para os indivíduos são supostamente medidos pela carga de estresse que é tanto física como psicológica (Munck-Ulfsfält et ál., 2003; . Saurin e Ferreira, 2009). 0 segundo construto P0, é definido como a forma que o trabalho está estruturado, distribuído, processado e supervisionado (Dixon et ál., 2009). A sua adoção integral pode depender de muitos fatores, tais como estilo de gestão, o tipo de produto ou serviço (Fettermann et ál., 2012; Echeveste et ál., 2017), características da força de trabalho, o nível e tipo de tecnologia de processos e condições de mercado (Carayon e Smith, 2000; Carayon, 2006; Guimarães et ál., 2015; Fettermann et ál., 2017; Calegari et ál., 2018).

Tabela 1

\section{Práticas ST na literatura}

\begin{tabular}{|c|c|c|c|c|c|c|c|c|c|c|c|c|c|c|c|}
\hline Práticas ST & (1) & (2) & (3) & $(4)$ & (5) & (6) & (7) & (8) & (9) & (10) & (11) & (12) & (13) & (14) & (15) \\
\hline $\begin{array}{l}\text { ST1-Sistemas de } \\
\text { comunicação de informação }\end{array}$ & $x$ & $x$ & & $x$ & $x$ & $x$ & $x$ & $x$ & $x$ & & & & & $x$ & $x$ \\
\hline $\begin{array}{l}\text { ST2- Resolução de problemas } \\
\text { e divulgação de indicadores }\end{array}$ & & & $x$ & & & $x$ & & & & & & $x$ & $x$ & & $x$ \\
\hline $\begin{array}{l}\text { ST3- Sobrecarga para } \\
\text { atingimento de metas }\end{array}$ & & & & $x$ & & & $x$ & $x$ & $x$ & $x$ & & & & & \\
\hline $\begin{array}{l}\text { ST4-Gerenciamento de } \\
\text { recursos humanos }\end{array}$ & & & & & $x$ & & & & & & & & & $x$ & $x$ \\
\hline $\begin{array}{l}\text { ST5- Utilização de conceitos } \\
\text { ergonomicos para o projeto } \\
\text { dos postos de trabalho }\end{array}$ & & $x$ & $x$ & $x$ & $x$ & $x$ & $x$ & $x$ & $x$ & $x$ & $x$ & $x$ & $x$ & $x$ & \\
\hline $\begin{array}{l}\text { ST6- Postos de trabalhos } \\
\text { adequados aos } \\
\text { colaboradores }\end{array}$ & & $x$ & $x$ & $x$ & $x$ & $x$ & $x$ & $x$ & $x$ & $x$ & $x$ & $x$ & $x$ & & \\
\hline $\begin{array}{l}\text { ST7- Reconhecimento } \\
\text { e recompensa para os } \\
\text { colaboradores }\end{array}$ & & & & & $x$ & & $x$ & $x$ & $x$ & & $x$ & & & & \\
\hline ST8- Trabalho em equipe & & & & & $x$ & $x$ & & $x$ & $x$ & & $x$ & $x$ & & & $x$ \\
\hline
\end{tabular}




\begin{tabular}{|c|c|c|c|c|c|c|c|c|c|c|c|c|c|c|c|}
\hline Práticas ST & (1) & (2) & (3) & $(4)$ & (5) & (6) & (7) & (8) & (9) & (10) & (11) & (12) & (13) & (14) & (15) \\
\hline $\begin{array}{l}\text { ST9- Definição de metas } \\
\text { claras }\end{array}$ & & & & & $x$ & & & $x$ & & & $x$ & & $x$ & $x$ & \\
\hline $\begin{array}{l}\text { ST10-Clareza nas } \\
\text { definições das funções dos } \\
\text { colaboradores }\end{array}$ & & $x$ & & $x$ & $x$ & & $x$ & $x$ & & & $x$ & $x$ & $x$ & & \\
\hline $\begin{array}{l}\text { ST11- Utilização de altertas } \\
\text { de riscos }\end{array}$ & & & $x$ & $x$ & & & & & $x$ & $x$ & $x$ & $x$ & $x$ & & $x$ \\
\hline $\begin{array}{l}\text { ST12- Busca de um bom } \\
\text { clima organizacional }\end{array}$ & $x$ & & $x$ & $x$ & $x$ & $x$ & $x$ & $x$ & $x$ & $x$ & $x$ & $x$ & $x$ & $x$ & $x$ \\
\hline $\begin{array}{l}\text { ST13- Busca da saúde e } \\
\text { segurança dos colaboradores }\end{array}$ & $x$ & & $x$ & $x$ & $x$ & $x$ & $x$ & $x$ & $x$ & $x$ & $x$ & $x$ & $x$ & $x$ & $x$ \\
\hline $\begin{array}{l}\text { ST14- Balanceamento entre } \\
\text { qualidade, escopo, tempo } \\
\text { e custo }\end{array}$ & $x$ & & & $x$ & $x$ & & $x$ & $x$ & $x$ & $x$ & $x$ & $x$ & $x$ & $x$ & \\
\hline $\begin{array}{l}\text { ST15- Prevenção e redução } \\
\text { de acidentes }\end{array}$ & $x$ & & $x$ & $x$ & $x$ & $x$ & $x$ & $x$ & $x$ & $x$ & $x$ & & & $x$ & $x$ \\
\hline $\begin{array}{l}\text { ST16-Valorização } \\
\text { do treinamento dos } \\
\text { colaboradores }\end{array}$ & $x$ & & & $x$ & $x$ & $x$ & $x$ & $x$ & $x$ & $x$ & $x$ & $x$ & & & $x$ \\
\hline $\begin{array}{l}\text { ST17- Utilização de } \\
\text { recomendações ergonomicas }\end{array}$ & $x$ & & & $x$ & $x$ & $x$ & $x$ & $x$ & & & $x$ & $x$ & & & $x$ \\
\hline $\begin{array}{l}\text { ST18-Regulamentação } \\
\text { de questões técnicas, } \\
\text { organizacionais e humanas }\end{array}$ & $x$ & & $x$ & $x$ & $x$ & $x$ & $x$ & $x$ & $x$ & $x$ & $x$ & $x$ & & $x$ & $x$ \\
\hline
\end{tabular}

(1) Hoffmesiter et al. (2015); (2) Haug (2015); (3) Azadeh et al. (2014); (4) Karsh et al. (2014); (5) Guimarães et al. (2014); (6) Asadzadeh et al. (2013); (7) Dul \& Neumann (2009); (8) Saurin \& Ferreira (2009); (9) Guimarães et al. (2015); (10) Koukoulaki (2014); (11) Koukoulaki (2010); (12) Zare et al. (2016); (13) Thun et al. (2011); (14) Pavlovic-Veselinovic et al. (2016); (15) Kang et al. (2016).

Fonte: elaboração propia dos autores.

Pesquisas e práticas no campo dos sistemas de ST demonstraram que considerando-se apenas um pequeno número de fatores de trabalho, pode ser enganosa e ineficiente a solução de problemas de projeto de trabalho (Zare et ál., 2016). No entanto, sistemas ST realmente enfatizam uma abordagem em que todos os elementos do sistema de trabalho devem ser considerados, a fim de melhorar o desempenho, saúde e qualidade (Asadzadeh et ál., 2013; Kang et ál., 2016). Por isso, propomos que os efeitos das práticas ST, indicados pelos fatores de PT e P0, influenciam positivamente na qualidade operacional (sucatas e retrabalhos) e saúde dos trabalhadores. Para examinar melhor isso, propomos a Proposição 1. 
Proposição 1: Práticas Socio-técnico, combinadas em construtos de PT e PO, estão associadas positivamente com o desempenho de qualidade e saúde dos trabalhadores.

\subsection{O efeito moderador JIT}

Um dos principais pilares do Sistema Toyota de Produção é o sistema de produção JIT (Womack e Jones, 2010), que visa idealmente a produção apenas do que o cliente quer e quando ele precisa (Liker, 2006). A implementação do JIT é cada vez mais visto como uma forma vital para a fabricação nas organizações, aumentando a sua competitividade. Além disso, há um conjunto de práticas LM, que têm sido extensivamente estudadas e empiricamente validadas como parte do JIT; são os seguintes: i) tempo de ciclo, ii) sistema puxado, iii) o fluxo contínuo, iv) o nivelamento da produção e (v) reposição do material (Shah e Ward, 2003; Shah e Ward, 2007; Netland e Ferdows, 2014).

Além de melhorar o desempenho operacional, algumas evidências indicam que a adoção do JIT pode impactar outros aspectos organizacionais (Tortorella et ál., 2016b). Evidências contraditórias sobre o efeito da adoção de práticas JIT sobre fatores humanos são encontrados na literatura. Por um lado, estudos anteriores relatam que o JIT abraça um modelo de produção que respeita a força humana e positivamente emprega conceitos de fatores humanos ao longo de suas atividades de melhoria (Plonka, 1997; Eklund, 2000; Saurin e Ferreira, 2009; Sharma, 2012). Eles argumentam que a adoção do JIT tende a melhorar a produção e o sistema de produção a partir da perspectiva do fluxo de material e de informações; durante a preparação da força de trabalho para responder com agilidade e eficácia às variações de demanda dos clientes (Jackson e Martin, 1996; Maia et ál., 2012).

Por outro lado, a integração de práticas JIT solicita um maior nível de auto-organização das tarefas da força de trabalho, o que exige equipes de produção para desenvolver uma base de competência coletiva que permite a resolução de problemas e lida com maiores graus de mudança (Gunasekaran et ál., 1998; Rahul et ál., 2014). Este aumento da demanda pode não ser sempre útil para o desempenho dos empregados, especialmente se os funcionários apresentam uma capacidade inferior cognitiva e de aprendizagem (Drews et ál., 2007; Eren, 2009; Koukoulaki, 2014). Os funcionários devem lidar com a incerteza e ter maior autonomia na resposta a eventos inesperados, também devem resolver problemas que possam causar pressões prejudiciais para eles, afetando tanto a qualidade e a produtividade do desempenho (Langstrand, 2012). Além disso, a realização de um sistema de produção JIT engloba os tempos de ciclo mais apertados, em 
que os tempos ociosos existentes são mitigados, aumentando potencialmente a fadiga dos operadores e exigências físicas (Womack et ál., 2009; Silva et ál., 2016).

A literatura complementar sobre sistemas ST assume que os indivíduos podem ser agentes não confiáveis propenso a erros, e, provavelmente, resistentes a mudanças (Blaikie et ál., 2014; Zare et ál., 2016), dificultando implicitamente a aplicação de abordagens de mudança, como o JIT. Assim, tendo em conta os argumentos acima mencionados, pode parecer que a adoção simultânea de JIT e práticas ST são mais susceptíveis de influenciar negativamente o desempenho da qualidade e da saúde dos trabalhadores, embora a evidência empírica para apoiar essa suposição ainda é escassa e contraditória. Portanto, para melhor investigar isso, nós formulamos a Proposição 2.

Proposição 2: A adoção de práticas just-in-time moderam negativamente a relação entre práticas sócio-técnicos e o desempenho da qualidade e da saúde dos trabalhadores.

\section{Método}

\subsection{A seleção de amostras e as características}

Os critérios de seleção da amostra de empresas foram as seguintes: (a) incluir líderes de empresas localizadas em uma região específica do país, neste caso, o Sul do Brasil, de forma a reduzir os efeitos do ambiente externo (cultura regional e desenvolvimento sócio-económico), uma vez que isso seria relativamente homogêneo dentro da amostra; (b) incluir empresas de diferentes setores industriais devido ao LM ter vindo a se expandir ao longo de muitos tipos de empresas nos últimos anos; e (c) os entrevistados devem ter experiência em lean e um papel de liderança na empresa. A escolha não aleatória das empresas para pesquisas e na busca de empresas que já são conhecidas para os investigadores, é uma estratégia comumente usada em outros estudos sobre LM (Saurin et ál., 2010; Boyle et ál., 2011; Tortorella et ál., 2016a). Por exemplo, Shah e Ward (2007) utilizou uma amostra com participantes escolhidos de cursos e eventos de treinamento quando realizou uma pesquisa sobre LM, uma vez que era necessário que os entrevistados tivessem experiência no assunto. 0 estudo de Kull et ál. (2014) sugere que a cultura nacional poderia influenciar a implementação de práticas enxutas. Por conseguinte, uma única localização geográfica também aumenta a homogeneidade da amostra. Além disso, embora a implementação LM seja geralmente associada com alto volume e fabricantes de peças discretas, a difusão de práticas em todo o especto industrial é desconhecido (Tortorella et ál., 2015). 
Os questionários foram enviados por e-mail a 523 ex-alunos dos cursos de educação executivo sobre lean oferecido por uma grande universidade brasileira desde 2014. A primeira mensagem de e-mail contendo os questionários foi enviada em junho de 2015, e dois acompanhamentos foram enviados nos meses seguintes. A amostra final foi composta de 144 respostas válidas (o que representa uma taxa de resposta de 27,53\%); a demografia dos entrevistados é apresentada na Tabela 2. A maioria dos entrevistados eram de grandes empresas (74,8\%); a maioria das empresas pertenciam ao setor de autopeças $(44,5 \%)$. Finalmente, quanto à experiência lean dentro das empresas, a maioria das empresas $(57,7 \%)$ apresentaram mais de 2 anos de implementação LM.

\section{Tabela 2}

Características da amostra

\begin{tabular}{|c|c|}
\hline & Percentual (\%) \\
\hline Pequena & 6,5 \\
\hline Média & 18,7 \\
\hline Grande & 74,8 \\
\hline Setor & Percentual \\
\hline Autopeças automotivas & 44,5 \\
\hline Alimentos & 28,2 \\
\hline Plásticos & 10,9 \\
\hline Metalúrgica & 5,5 \\
\hline Têxtil & 3,6 \\
\hline Embalagem & 2,7 \\
\hline Química & 1,8 \\
\hline Outros & 2,7 \\
\hline Implementação Lean & Percentual \\
\hline Menos que 2 anos & 42,3 \\
\hline Mais que 2 anos & 57,7 \\
\hline
\end{tabular}

Fonte: elaboração propia dos autores.

\subsection{Questionário e coleta de dados}

0 questionário tinha quatro partes: a primeira teve como objetivo recolher informações demográficas dos entrevistados e suas empresas, tais como tamanho, setor e experiência lean dos entrevistados. Usamos tamanho e tempo de implementação LM como variável de controle em nosso modelo de regressão linear. A segunda parte destina-se a avaliar o grau de adoção das dezoito práticas ST (ver Tabela 1). 0s respondentes foram 
convidados a indicar o nível de aprovação de acordo com uma escala de Likert de 5 pontos, variando de 1 (não aplicado) a 5 (totalmente adotado). A terceira parte destina-se a medir o grau de adoção das cinco práticas JIT acima mencionadas. A mesma escala de Likert foi utilizado para indicar o nível de adoção de práticas JIT. Finalmente, a quarta parte do questionário compreendeu a identificação do grau de variação da qualidade e desempenho de saúde dos trabalhadores ao longo dos últimos cinco anos, dentro das respondentes empresas. Da mesma forma, uma escala de Likert de 5 pontos foi usado para indicar essa variação de desempenho, em que 1 indicaram "piorou significativamente", e 5 "melhorou significativamente".

Em relação à avaliação do nível de implementação de práticas ST e JIT e a variação de desempenho, testamos o viés da não resposta como proposto por Armstrong e Overton (1977), utilizando o teste de Levene para igualdade de variâncias e o teste de igualdade das médias entre o inicais (respondentes do primeiro e-mail enviado) e tardios (respondentes das duas continuações) respondentes. Os resultados indicaram não haver diferenças significativas $(p<0,05)$ em meio a variação dos dois grupos. Assim, não há nenhuma evidência estatística de que a amostra é significativamente diferente do resto da população para todas as variáveis.

\subsection{Análise de dados}

Tabela 3 apresenta a CFA para as práticas de ST. As dezoito práticas foram combinadas em dois construtos. Por exemplo, todas as práticas relacionadas com a concepção e melhoria da ergonomia das estações de trabalho foram combinadas para formar PT. A lógica subjacente é que PT desempenha um papel fundamental na realização de uma vida de trabalho mais saudável e uma integração com os processos e exigências dos trabalhadores. 0 construto $0 \mathrm{P}$ compreende práticas que podem ser identificadas por seu foco em melhorar os aspectos que são mais amplos do que a própria (por exemplo, sistema de comunicação e informação, resolver o problema da exposição de indicadores, a sobrecarga para cumprimento de metas, a gestão de rotatividade de pessoas) estação de trabalho relacionada a uma gestão eficiente de aspectos sócio-técnicos implícitos às rotinas organizacionais (Blaikie et ál., 2014).

Cada um dos construtos foi formado pela soma das pontuações para cada prática incluída no construto das respostas das empresas. Todas as 18 práticas ST foram inseridas para PCA (análise de componentes principais) e a rotação varimax foi utilizada para extrair componentes ortogonais, e dois componentes foram extraídos (PT e P0). Assim, os 
construtos foram empiricamente validados usando PCA com a rotação varimax e a análise de confiabilidade (alpha de Cronbach), como mostra a Tabela 3. Os dados foram analisados usando SPSS versão 23. Os resultados foram replicados usando rotação oblíqua como uma checagem de ortogonalidade e os componentes semelhantes foram extraídos. Além disso, a unidimensionalidade de cada componente foi verificada e confirmada pela aplicação de PCA no nível do componente. A avaliação da confiabilidade foi realizada com a determinação dos valores do alfa de Cronbach para cada componente, que depende do número de itens na escala e a correlação média entre os itens (Meyers et ál., 2006). Todos os componentes exibiram um alta de confiabilidade, com valores acima de 0,910. Finalmente, o valor de resposta para cada construto foi obtido através da média das práticas correspondentes incluídos no construto, ponderadas pelos respectivos pesos dos fatores de PCA. Os componentes de cargas variáveis obtidos (método de regressão Thurstone's) foram usados na regressão. Portanto, as cargas variáveis de P0 e PT foram introduzidos como novas variáveis independentes no modelo de regressão.

De forma análoga, em relação às práticas JIT, uma escala foi construída para a medida JIT baseado em PCA das cinco práticas acima mencionadas, e os fatores pontuação foram usados como variável moderadora. A Tabela 4 mostra que todas as cinco práticas carregam um fator, com um valor próprio de 3,13 explicando 62,68\% da variação. Além disso, o valor alfa de Cronbach obtido é 0,850 e o resultado para o teste de esfericidade de Bartlett foi significativo, uma vez que foi menor que 0,001 (Meyers et ál., 2006). Assim, assumimos que todas as cinco práticas são empiricamente relacionadas e representam uma dimensão única de JIT.

\section{Tabela 3}

PCA para validação dos construtos de práticas ST - Matriz de componentes rotacionados (varimax)

\begin{tabular}{|c|c|c|c|}
\hline Práticas ST & $\begin{array}{c}1 \\
\text { (Projeto do Trabalho PT) }\end{array}$ & $\begin{array}{c}2 \\
\text { (Práticas Organizacionais-P0) }\end{array}$ & Comunalidades \\
\hline $\mathrm{ST}_{5}$ & 0,651 & 0,319 & 0,525 \\
\hline $\mathrm{ST}_{6}$ & 0,756 & 0,268 & 0,644 \\
\hline $\mathrm{ST}_{8}$ & 0,623 & 0,504 & 0,643 \\
\hline $\mathrm{ST}_{11}$ & 0,667 & 0,430 & 0,629 \\
\hline $\mathrm{ST}_{12}$ & 0,750 & 0,353 & 0,688 \\
\hline $\mathrm{ST}_{13}$ & 0,751 & 0,362 & 0,695 \\
\hline $\mathrm{ST}_{14}$ & 0,640 & 0,466 & 0,626 \\
\hline $\mathrm{ST}_{15}$ & 0,638 & 0,486 & 0,643 \\
\hline
\end{tabular}




\begin{tabular}{|c|c|c|c|}
\hline Práticas ST & $\begin{array}{c}1 \\
\text { (Projeto do Trabalho PT) }\end{array}$ & $\begin{array}{c}2 \\
\text { (Práticas Organizacionais-P0) }\end{array}$ & Comunalidades \\
\hline $\mathrm{ST}_{17}$ & 0,819 & 0,251 & 0,734 \\
\hline $\mathrm{ST}_{18}$ & 0,711 & 0,469 & 0,725 \\
\hline $\mathrm{ST}_{1}$ & 0,443 & 0,700 & 0,686 \\
\hline $\mathrm{ST}_{2}$ & 0,330 & 0,748 & 0,669 \\
\hline $\mathrm{ST}_{3}$ & 0,174 & 0,747 & 0,589 \\
\hline $\mathrm{ST}_{4}$ & 0,420 & 0,663 & 0,616 \\
\hline $\mathrm{ST}_{7}$ & 0,352 & 0,666 & 0,568 \\
\hline $\mathrm{ST}_{9}$ & 0,367 & 0,714 & 0,644 \\
\hline $\mathrm{ST}_{10}$ & 0,520 & 0,640 & 0,680 \\
\hline $\mathrm{ST}_{16}$ & 0,456 & 0,595 & 0,561 \\
\hline Eigenvalues & 10,560 & 1,005 & \\
\hline Alfa de Cronbach & 0,938 & 0,910 & \\
\hline \% da variância & $58,66 \%$ & $5,58 \%$ & \\
\hline Cumulativo \% & & $62,68 \%$ & \\
\hline Bartlett teste de esferidade & & 0,000 & \\
\hline Kaiser-Meyer-0kin (KMO) & & 0,938 & \\
\hline
\end{tabular}

Fonte: elaboração propia dos autores.

\section{Tabela 4}

PCA para validar construto JIT - Matriz de componentes rotacionados (varimax)

\begin{tabular}{lcc}
\hline \multicolumn{1}{c}{ Práticas JIT } & Carga Fatorial & Comunalidades \\
\hline 1- Takt time & 0,745 & 0,554 \\
2- Sistema Puxado & 0,839 & 0,704 \\
3- Fluxo contínuo & 0,804 & 0,646 \\
4- Nivelamento da produção & 0,742 & 0,551 \\
5-Abastecimento de materiais & 0,824 & 0,678 \\
\hline Eigenvalue & 3,13 & \\
Alfa de Cronbach & 0,850 & \\
\% da variância & $62,68 \%$ & \\
Bartlett teste de esferidade & 0,000 & \\
Kaiser-Meyer-0kin (KM0) & 0,831 & \\
\hline
\end{tabular}

Fonte: elaboração propia dos autores.

Finalmente, uma terceira PCA foi realizada com relação ao desempenho, como mostra a Tabela 5. Ambos os indicadores (qualidade e saúde dos trabalhadores) foram combinados 
em um componente de desempenho, que foi usado mais tarde como variável dependente nos modelos de regressão por mínimos quadrados. 0 resultado para o teste de esfericidade de Bartlett foi significativo, uma vez que foi menor que 0,001 (Meyers et ál., 2006). 0 valor próprio do componente de desempenho foi superior a 1,0, e a variância explicada era maior do que 50\%, tal como recomendado pelo Tabachnick e Fidell (2013). Para fins de confiabilidade, o valor alfa de Cronbach é 0,640, indicando validade satisfatória (Hair et ál., 2006).

\section{Tabela 5}

PCA para validar o desempenho - matriz componente rodado

\begin{tabular}{lcc}
\multicolumn{1}{c}{ Indicadores } & Carga Fatorial & Comunalidades \\
\hline Saúde dos colaboradores & 0,859 & 0,737 \\
Qualidade (sucata e retrabalho) & 0,859 & 0,737 \\
\hline Eigenvalue & 1,47 & \\
Alfa de Cronbach & 0,640 & \\
$\%$ da variância & $73,74 \%$ & \\
Bartlett teste de esferidade & 0,000 & \\
Kaiser-Meyer-0kin (KM0) & 0,600 & \\
\hline
\end{tabular}

Fonte: elaboração propia dos autores.

Após estabelecer os componentes (independente, moderadores e variáveis dependentes), foi realizado um conjunto de modelos de regressão OLS para testar as proposições teóricas. 0 cálculo dos termos de interação (P0 x JIT e PT x JIT) foi obtido pelo produto das variáveis independentes padronizadas, como recomendado pelo Baron e Kenny (1986). Portanto, nossos resultados relataram os coeficientes não padronizados, uma vez que as escalas foram padronizados antes da análise, ou seja, os coeficientes não padronizados irão representar um efeito padronizado (Goldsby et ál., 2013). Além disso, o tamanho da empresa e o tempo de implementação LM foram incluídos como variáveis de controle no modelo. Ambas as variáveis foram classificadas em duas categorias. 0 tamanho da empresa foi codificado em grande: com mais de 500 empregados, e pequenas quando tem 500 ou menos empregados. Para o tempo de implementação LM, as empresas foram divididas em as que apresentam mais ou menos 2 anos, como evidenciado no estudo de Marodin et ál. (2016).

Testamos os pressupostos da distribuição normal, linearidade e homocedasticidade entre as variáveis independentes (preditora focal e moderadora) e as variáveis dependentes (desempenho) (Hair et ál., 2006). Além disso, os resíduos foram examinados a fim de 
confirmar a normalidade de distribuição do termo de erro. A linearidade foi testada com parcelas de regressão parcial para cada modelo. Finalmente, a homocedasticidade foi avaliada através da representação gráfica de resíduos padronizados contra o valor previsto e examinada visualmente.

\section{Resultados}

A Tabela 6 apresenta os modelos de regressão. 0 primeiro modelo investiga a relação entre as variáveis de controle (o tamanho da firma e o tempo de aplicação LM) e desempenho. 0 modelo 2 inclui a associação entre construtos de práticas ST (PT e P0) e desempenho, a fim de examinar a validade da Proposição 1. Finalmente, o modelo 3 adiciona o efeito das práticas JIT e seu efeito moderador sobre as práticas de ST (P0 x JIT e PT x JIT) para explicar a variação de desempenho, tal como indicado na Proposição 2. A variável dependente (desempenho) é mostrada nas colunas, enquanto que o controle e as variáveis independentes e o termo de interação estão localizados nas linhas. 0s fatores de inflação da variância (VIFs) nos modelos de regressão foram todos inferiores a 2,0, sugerindo que a multicolinearidade não foi uma preocupação.

Os resultados para o modelo 2 indicam que quase $30 \%$ da variância do desempenho é explicado de forma significativa $(p<0,001)$. A inclusão das variáveis independentes (PT e P0) no modelo de regressão melhora significativamente (mudança $F=19,887$; $p$ $<0,001$ ) a capacidade de previsibilidade do modelo em relação ao modelo 1 (variáveis de controle). Além disso, ambos os construtos ST (PT e P0) estão associados positivamente $(\beta=0,397$ e $\beta=0,286 ; p<0,001$, respectivamente) com a qualidade e saúde dos trabalhadores, confirmando totalmente a Proposição 1. Este resultado é consistente com os estudos de Carayon (2006), Koukoulaki (2010) e Asadzadeh et ál. (2013), que indica que o efeito da adoção de práticas ST em organizações pode levar a resultados positivos, contribuindo na qualidade, nos indicadores de saúde e de desempenho dos trabalhadores como um todo. Portanto, este resultado evidencia a importância de implementar adequadamente tanto os aspectos sociais e técnicos, e tratá-los de forma interdependente dentro do sistema de trabalho para fornecer os efeitos esperados.

Embora o modelo 3 parece explicar a variação de desempenho significativamente $(\mathrm{p}<$ $0,001)$, a inclusão dos termos de interação não parece melhorar significativamente a capacidade de desempenho quando comparados com o modelo 2. Esses resultados podem ser evidenciados pelos resultados para a melhoria da previsibilidade do modelo 3 sobre o modelo 2 (mudança de $F=0,647$; Sig. $F$ mudança $=0,526$ ), e , portanto, não vencem 
a Proposição 2. Portanto, não encontramos evidências empíricas para apoiar a Proposição 2 com base na amostra estudada , o que indica que a implementação simultânea de práticas JIT não põe em causa o efeito das práticas ST na qualidade e desempenho da saúde dos trabalhadores.

No geral, o modelo final adotado para o desempenho da qualidade e da saúde dos trabalhadores foi o modelo 2, sugerindo que ambos os conjuntos de construtos ST tem um efeito direto e positivo no desempenho, e não há nenhum efeito de moderação com a adoção do JIT. Esta descoberta é tanto coerente com os resultados a partir de Jackson e Martin (1996) e Toralla et ál. (2012), que afirmam que a implementação do JIT e de outras práticas LM não afeta diretamente o desempenho na saúde e na qualidade dos trabalhadores. Em oposição, os nossos resultados indicam que, se as práticas de ST são eficazes e implementadas de forma consistente dentro da organização, todos os potenciais efeitos colaterais da adoção JIT (por exemplo, pressão para a realização do cronograma de entrega, estabelecimento e controle dos processos de estabilização e normalização, a rápida e afirmativa resolução de problemas, etc.) não podem influenciar os níveis de qualidade e saúde dos trabalhadores. Este fato desmistifica as suposições e associações erradas que a implementação LM, denotada neste estudo pela adoção de práticas JIT, gera níveis mais elevados de exigências físicas, psicológicas e cognitivas.

\section{Tabela 6}

Resultados dos modelos de regressão por mínimos quadrados

\begin{tabular}{|c|c|c|c|}
\hline & Modelo 1 & Modelo 2 & Modelo 3 \\
\hline Porte da empresa & $0,276^{\star *}$ & $0,160^{\star \star}$ & $0,171^{\star}$ \\
\hline Tempo de LM implementação & 0,134 & 0,055 & 0,049 \\
\hline PT & & $0,397^{\star \star \star}$ & $0,360^{* * *}$ \\
\hline PO & & $0,286^{\star \star \star}$ & $0,258^{* *}$ \\
\hline JIT & & & 0,042 \\
\hline $\mathrm{PT}^{*} \mathrm{JIT}$ & & & $-0,071$ \\
\hline PO*JIT & & & $-0,045$ \\
\hline $\mathrm{F}$ & 6,078 & 14,548 & 8,168 \\
\hline Sig, & $0,003^{* *}$ & $0,000^{* \star *}$ & $0,000^{* \star \star}$ \\
\hline $\mathrm{R}^{2}$ ajustado & 0,070 & 0,297 & 0,285 \\
\hline F mudança & & 19,887 & 0,647 \\
\hline Sig F mudança & & $0,000^{\star \star *}$ & 0,526 \\
\hline
\end{tabular}

"significante a $10 \% /{ }^{* *}$ significante a $5 \% /{ }^{* * *}$ significante a $1 \%$.

Fontes: elaboração propia dos autores. 


\section{Conclusões}

Foi realizada uma pesquisa baseada em dados de 144 empresas do Sul do Brasil que são submetidas a uma implementação Lean Manufacturing (LM), a fim de responder às seguintes questões de pesquisa: (i) como é que a adoção do JIT modera a relação entre as práticas ST e o desempenho da qualidade e da saúde dos trabalhadores? Os dados foram analisados inicialmente através de um CFA, a fim de confirmar os construtos de ambas as práticas ST e JIT e uma construção de desempenho. Mais tarde, os três modelos de regressão foram gerados mediante a aplicação de uma regressão OLS indicando a importância das relações estudadas.

A contribuição deste estudo é dupla. Em primeiro lugar, os resultados mostram que os construtos de práticas ST (Projeto de Trabalho e Práticas Organizacionais) considerados neste trabalho estão associados positivamente com o desempenho de qualidade e saúde dos trabalhadores nas empresas estudadas. Assim, confirmou-se que o uso das dezoito práticas ST contribui para melhorar o desempenho em sucatas e retrabalho, bem como melhorar a qualidade de vida no trabalho mitigando o absentismo e a rotatividade. Este resultado é compativel com os resultados de estudos anteriores que enfatizam a importância de implementar plenamente as práticas ST através de um projeto integrado; portanto, evitando a adoção de aspectos unilaterais que possam prejudicar a realização dos potenciais benefícios.

Em segundo lugar, a adoção simultânea de práticas JIT, como tempo do ciclo, sistema de fluxo contínuo, o nivelamento da produção e reposição puxada de material, não parece influenciar significativamente a associação entre práticas ST e qualidade e saúde dos trabalhadores. Portanto, nossos resultados sugerem que se as práticas de ST estão adequadamente no lugar, a adoção do JIT não pode prejudicar fisicamente empregados ou o seu desempenho em detrimento da qualidade. Em termos práticos, fornecemos evidências empíricas para os gestores e profissionais que a implementação do LM (restrito aqui pela adoção de práticas JIT) não ocorre à custa de prejudicar a saúde do trabalhador ou que afetem as questões relacionadas com os parâmetros de qualidade e controle de processo. Na verdade, os resultados mostram que o efeito das práticas de ST pode prevalecer para melhorar tal desempenho (qualidade e saúde dos trabalhadores), destacando que ambos os aspectos técnicos e sociais da organização deve ser dirigida principalmente a fim de proporcionar um ambiente organizacional adequado para a implementação de práticas LM específicas e seus construtos, como JIT. 
Embora o tamanho da amostra possa ser considerada pequena para levantamentos empíricos, todos os testes foram feitos com significância. Essas interações que resultaram em um efeito significativo, aceitou o tamanho da amostra com os respectivos graus de liberdade necessários. Como desvantagens, nossos resultados são limitados pelas características da amostra (isto é, a localização como sul do Brasil) e pode ser particularizado a este caso. Assim, ampliando a amostra a outras regiões e países, diversificando a outros setores de produção e incluindo outros construtos de práticas LM além do JIT, são sugestões válidas para futuras pesquisas. Particularmente, seria interessante incluir empresas de outros países com economias emergentes, como a China e a Índia, e também de países desenvolvidos, como EUA e da Europa, para comparar o efeito moderador do LM sobre a relação entre práticas e desempenho ST. Além disso, os padrões de implementação das práticas ST ao longo do tempo poderia ser mais investigada a fim de produzir estruturas melhores para as empresas ao avaliar as suas iniciativas de melhoria.

\section{Referências}

Arezes, P., Carvalho, D., \& Alves, A. (2010). Threats and opportunities for workplace ergonomics in lean environments. In 17th International Annual EurOMA Conference-Managing Operations in Service Economics. EurOMA.

Arezes, P., Dinis-Carvalho, J., \& Alves, A. (2015). Workplace ergonomics in lean production environments: A literature review. Work, 52(1), 57-70. D0I: 10.3233/W0R-141941.

Armstrong, J., \& Overton, S. (1997). Estimating nonresponse bias in mail surveys. Journal of Marketing Research, 14(3), 396-402.

Asadzadeh, S., Azadeh, A., Negahban, A., \& Sotoudeh, A. (2013). Assessment and improvement of integrated HSE and macro-ergonomics factors by fuzzy cognitive maps: The case of a large gas refinery. Journal of Loss Prevention in the Process Industries, 26(6), 1015-1026.

Azadeh, A., Rouzbahman, M., Saberi, M., \& Valianpour, F. (2014). An adaptive algorithm for assessment of operators with job security and HSEE indicators. Journal of Loss Prevention in the Process Industries, 31, 26-40.

Bäckstrand, G., Bergman, C., Hogberg, D., \& Moestam, L. (2013). Lean and its impact on workplace design. Proceedings of NES 45th Nordic Ergonomics \& Human Factors Society Conference, Iceland.

Baron, R., \& Kenny, D. (1986). The moderator-mediator variable distinction in social psychological research: Conceptual, strategic, and statistical considerations. Journal of Personality and Social Psychology, 51(6), 1173-1182. http://dx.doi.org/10.1037/0022-3514.51.6.1173

Beevis, D. (2003). Ergonomics: cost and benefits revisited. Applied Ergonomics, 34, 491-496. 
Blaikie, P., Cannon, T., Davis, I., \& Wisner, B. (2014). At risk: Natural hazards, people's vulnerability and disasters. Routledge.

Boyle, T., Scherrer-Ratheje, M., \& Stuart, I. (2011). Learning to be lean: The influence of external information sources in lean improvements. Journal of Manufacturing Technology Management, 22(5), 587-603. https://doi.org/10.1108/17410381111134455

Calegari, L., Barbosa, J., Marodin, G., \& Fettermann, D. (2018). A conjoint analysis to consumer choice in Brazil: Defining device attributes for recognizing customized foods characteristics. Food Research International, 109, 1-13. D0I: 10.1016/j.foodres.2018.03.080

Carayon, P. (2006). Human factors of complex sociotechnical systems. Applied Ergonomics, $37(4), 525-535$.

Carayon, P., \& Smith, M. (2000). Work organization and ergonomics. Applied Ergonomics, 31(6), 649-662.

Clegg, C. (2000). Sociotechnical principles for system design. Applied Ergonomics, 31, 463-477.

Dixon, S., Theberge, N., \& Cole, D. (2009). Sustaining management commitment to workplace health programs: The case of participatory ergonomics. Industrial Relations, 64(1), 50-74.

Drew, F., Picciano, P., Agutter, J., Syroid, N., Westenskow, D., \& Strayer, D. (2007). Development and evaluation of a just-in-time support system. Human Factors, 49(3), 543-551.

Dul, J., \& Neumann, W. (2009). Ergonomics contributions to company strategies. Applied Ergonomics, 40(4), 745-752.

Echeveste, M., Rozenfeld, H., \& Fettermann, D. (2017). Customizing practices based on the frequency of problems in new product development process. Concurrent Engineering, 25(3), p. 1063293X1668615-265. D0I: 10.1177/1063293X16686154

Eklund, J. (2000). Development work for quality and ergonomics. Applied Ergonomics, 31(6), 641-648

Eren, T. (2009). Human and machine effects in a just-in-time scheduling problem. Human Factors and Ergonomics in Manufacturing, 19(4), 294-299.

Ferreira, L., \& Gurgueira, G. (2013). Ergonomia como fator econômico no pensamento Enxuto: uma análise crítica bibliográfica. Gepros: Gestão da Produção, Operações e Sistemas, 8(3), 39-49.

Fettermann, D., Cavalcante, C., Almeida, T, \& Tortorella, G. (2018). How does Industry 4.0 contribute to Operations Management? Journal of Industrial and Production Engineering, 35(4), 255-268. https://doi.org/10.1080/21681015.2018.1462863

Fettermann, D., Echeveste, M., \& Ten Caten, C. (2012). When and How to use the online configurator in the Automobile Industry. Revista IEEE América Latina, 10(6), 2331-2341. D0I:10.1109/TLA.2012.6418140. 
Fettermann, D., Echeveste, M., \& Tortorella, G. (2017). The benchmarking of the use of toolkit for mass customization in the automobile industry. Benchmarking, 24(6), 1767-1783. DOI: 10.1108/BIJ-01-2016-0002

Genaidy, A., \& Karwowski, W. (2003). Human performance in lean production environment: Critical assessment and research framework. Human Factors and Ergonomics in Manufacturing \& Service Industries, 13(4), 317-330.

Goldsby, T., Knemeyer, A., Miller, J., \& Wallenburg, C. (2013). Measurement and moderation: Finding the boundary conditions in logistics and supply chain research. Journal of Business Logistics, 34(2), 109-116.

Guimarães, L., Anzanello, M., Ribeiro, J., \& Saurin, T. (2015). Participatory ergonomics intervention for improving human and production outcomes of a Brazilian furniture company. International Journal of Industrial Ergonomics, 49, 97-107.

Guimarães, L., Ribeiro, J., Renner, J., \& De Oliveria, P. (2014). Worker evaluation of a macroergonomic intervention in a Brazilian footwear company. Applied Ergonomics, 45(4), 923-935.

Gunasekaran, A., Goyal, S., Martikainen, T., \& Yli-0lli, P. (1998). A conceptual framework for the implementation of zero inventory and just-in-time manufacturing concepts. Human Factors and Ergonomics in Manufacturing, 8(1), 63-78.

Hagg, G. (2003). Corporate initiatives in ergonomics: an introduction. Applied Ergonomics, 34, 3-15.

Hair, J., Tatham, R., Anderosn, R., \& Black, W. (2006). Multivariate data analysis. Pearson Prentice Hall, Upper Saddle River, NJ.

Haug, A. . (2015). Work instruction quality in industrial management. International Journal of Industrial Ergonomics, 50, 170-177. http://dx.doi.org/10.1016/j.ergon.2015.09.015

Hoffmeister, K., Gibbons, A., Schwatka, N., \& Rosecrance, J. (2015). Ergonomics climate assessment: a measure of operational performance and employee well-being. Applied Ergonomics, 50, 160-169. D0I: 10.1016/j.apergo.2015.03.011

Jackson, P., \& Martin, R. (1996). Impact of just-in-time on job content, employee attitudes and well-being: a longitudinal study. Ergonomics, 39(1), 1-16.

Jaworek, M., Marek, T., Karwowski, W., Andrzejczak, C., \& Genaidy, A. (2010). Burnout syndrome as a mediator for the effect of work-related factors on musculoskeletal complaints among hospital nurses. International Journal of Industrial Ergonomics, 40(3), 368-375.

Joseph, B. (2003). Corporate ergonomics programme at Ford Motor Company. Applied Ergonomics, 34, 23-28.9.

Kang, J., Zhang, J., \& Gao, J. (2016). Improving performance evaluation of health, safety and environment management system by combining fuzzy cognitive maps and relative degree analysis. Safety Science, 87, 92-100. D0I: 10.1016/j.ssci.2016.03.023 
Karsh, B., Waterson, P., \& Holden, R. (2014). Crossing levels in systems ergonomics: a framework to support 'mesoergonomic' inquiry. Applied Ergonomics, 45(1), 45-54. D0I:10.1016/j. apergo.2013.04.021

Karwowski, W., Salvendy, G., Badham, R., et ál. (1994). Integrating people, organization, and technology in advanced manufacturing: a position paper based on the joint view of industrial managers, engineers, consultants, and researchers. International Journal of Human Factors in Manufacturing, 4(1), 1-19.

Koukoulaki, T. (2010). New trends in work environment. New effects on safety. Safety Science, 48(8), 936-942. D0I:10.1016/j.ssci.2009.04.003.

Koukoulaki, T. (2014). The impact of lean production on musculoskeletal and psychosocial risks: An examination of sociotechnical trends over 20 years. Applied Ergonomics, 45, 198-121.

Langstrand, J. (2012). Exploring organizational translation: a case study of changes toward lean production. Doctoral Thesis, Linköping University, Department of Management and Engineering, Quality Technology and Management.

Liker, J. (2006). The Toyota way fieldbook. New York: McGrawHill.

Liker, J., \& Hoseus, M. (2008). Toyota culture: The heart and soul of the Toyota way. New York: McGrawHill.

Maia, L., Alves, A., \& Leão, C. (2012). Design of a lean methodology for an ergonomic and sustainable work environment in textile and garment industry. Proceedings of ASME International Mechanical Engineering Congress and Exposition, Houston, Texas, USA.

Marodin, G., Frank, A., Tortorella, G., \& Saurin, T. (2016). Contextual factors and Lean Production implementation in the Brazilian automotive supply chain. Supply Chain Management: An International Journal, 21(4). DOI: 10.1080/14783363.2017.1308221

Meyers, L., Gamst, G., \& Guarino, A. (2006). Applied Multivariate Research. Sage Publications, Thousand Oaks.

Molleman, E., \& Broekhuis, M. (2001). Sociotechnical systems: towards an organizational learning approach. Journal of Engineering Technology Management, 18, 271-294.

Munck-Ulfsfalt, U., Falck, A., Forsberg, A., Dahlin, C., \& Eriksson, A. (2003). Corporate ergonomics programme at Volvo car corporation. Applied Ergonomics, 34(1), 17-22.

Netland, T., \& Ferdows, K. (2014). What to expect from a corporate lean program. MIT Sloan Management Review, 55(3), 83-89.

Pavlovic-Veselinovic, S., Hedge, A., \& Veselinovic, M. (2016). An ergonomic expert system for risk assessment of work-related musculo-skeletal disorders. International Journal of Industrial Ergonomics, 53, 130-139.

Plonka, F. (1997). Developing a lean and agile work force. Human Factors and Ergonomics in Manufacturing \& Services Industries, 7(1), 11-20. 
Rahul, P., Ravindran, M., Sarvesh, S., Rajesh, D. (2014). Cycle time reduction in assembly line through layout improvement, ergonomics analysis and lean principles. International Journal of Applied Science and Engineering Research, 3(2), 455-463.

Saurin, T., Ferreira, C. (2009). The impacts of lean production on working conditions: A case study of a harvester assembly line in Brazil. International Journal of Industrial Ergonomics, 39, 403-412.

Saurin, T., Ribeiro, J., Marodin, G. (2010). Identification of research opportunities based on a survey on lean production implementation conducted in Brazilian and foreign companies. Gestão \& Produção, 17(4), 829-841.

Seppälä, P., \& Klemola, S. (2004). How do employees perceive their organization and job when companies adopt principles of lean production? Human Factors and Ergonomics in $\mathrm{Ma}$ nufacturing \& Service Industries, 14(2), 157-180.

Shah, R., \& Ward, P. (2003). Lean manufacturing: context, practice bundles, and performance. Journal of Operations Management, 21, 129-149.

Shah, R., \& Ward, P. (2007). Defining and developing measures of lean production. Journal of Operations Management, 25(4), 785-805.

Sharma, R. (2012). Conceptual framework for improving business performance with lean manufacturing and successful human factors interventions-a case study. International Journal for Quality Research, 6(3), 259-270.

Silva, M., Tortorella, G., \& Amaral, F. (2016). Psychophysical demands and perceived workload, an ergonomics standpoint for lean production in assembly cells. Human Factors and Ergonomics in Manufacturing \& Service Industries, (forthcoming).

Tabachnick, B., \& Fidell, L. (2013). Using Multivariate Statistics, $5^{\text {a }}$ ed., Pearson, Upper Saddle River, NJ.

Thun, J., Lehr, C., \& Bierwirth, M. (2011). Feel free to feel comfortable: An empirical analysis of ergonomics in the German automotive industry. International Journal of Production Economics, 133(2), 551-561. D0I:10.1016/j.ijpe.2010.12.017

Toralla, P., Falzon, P., \& Morais, A. (2012). Participatory design in lean production: Which contribution from employees? For what end? Work, 41, 2706-2712. D0I: 10.3233/WOR2012-0514-2706

Tortorella, G., Viana, S., \& Fettermann, D. (2015). Learning cycles and focus groups: A complementary approach to the $A 3$ thinking methodology Learning Organization, 22(4), 229240. https://doi.org/10.1108/TL0-02-2015-0008

Tortorella, G., Marodin, G., Miorando, R., \& Seidel, A. (2015). The impact of contextual variables on learning organization in firms that are implementing lean: A study in Southern Brazil. International Journal of Advanced Manufacturing Technology, 78(9-12), 1879-1892. 
Tortorella, G., Marodin, G., Fettermann, D., \& Fogliatto, F. (2016a). Relationships between lean product development enablers and problems. International Journal of Production Research, 54(10), 2837-2855. https://doi.org/10.1080/00207543.2015.1106020

Tortorella, G., Vergara, L., \& Ferreira, E. (2016b). Lean manufacturing implementation: An assessment method with regards to socio-technical and ergonomics practices adoption. International Journal of Advanced Manufacturing Technology, (forthcoming).

Wilson, R. (2005). Guarding the line: Score big by planning for worker safety while you implement lean. Industrial Engineer, 37(4), 46-50.

Womack, J., \& Jones, D. (2010). Lean thinking: Banish waste and create wealth in your corporation, 2a. ed. New York: Simon and Schuster.

Womack, S., Armstrong, T., \& Liker, J. (2009). Lean job design and musculoskeletal disorder risk: a two plant comparison. Human Factors and Ergonomics in Manufacturing \& Service Industries, 19(4), 279-293. D0I: 10.1002/hfm.20159

Zare, M., Croq, M., Hossein-Arabi, F., Brunet, R., \& Roquelaure, Y. (2016). Does ergonomics improve product quality and reduce costs? A review article. Human Factors and Ergonomics in Manufacturing \& Service Industries, 26(2), 205-223. https://doi.org/10.1002/hfm.20623 